\title{
Generation of Magnetic Fields by a Gravitomagnetic Plasma Battery
}

\author{
Ramon Khanna \\ Landessternwarte, Königstuhl, D-69117 Heidelberg, Germany \\ rkhanna@lsw.uni-heidelberg.de
}

9 September 2021

\begin{abstract}
The generation of magnetic fields by a battery, operating in an ion-electron plasma around a Kerr black hole, is studied in the $3+1$ split of the Kerr metric. It is found that the gravitomagnetic contributions to the electron partial pressure are able to drive currents. The strength of the equilibrium magnetic field should be higher than for the classical Biermann battery, which is found to operate in this relativistic context as well, since the gravitomagnetic driving terms can less easily be quenched than the classical ones. In axisymmetry the battery can induce only toroidal magnetic fields. Once a toroidal magnetic field is present, however, the coupling of gravitomagnetic and electromagnetic fields generates a poloidal magnetic field even in axisymmetry (Khanna \& Camenzind 1996a,b). A rotating black hole, embedded in plasma, will therefore always generate toroidal and poloidal magnetic fields.
\end{abstract}

Key words: black hole physics - MHD - plasmas - relativity

\section{INTRODUCTION}

In the year 1950 Ludwig Biermann published a paper 'On the Origin of Magnetic Fields on Stars and in Interstellar Space'. He showed that currents must flow in a plasma, if its effective acceleration possesses a rotational part and thus charge separation due to the electron partial pressure can not be balanced by an electrostatic field. In the case of an initially non-magnetized, differentially rotating star with no meridional circulation, this battery would generate magnetic fields of the order kGauss. Mestel \& Roxburgh (1962) revisited 'Biermann's battery', considering the effects of meridional circulation and the presence of a weak poloidal magnetic field. They concluded that, due to Lorentz forces modifying the plasma rotation, the magnetic field will saturate at much lower field strength than it would due to Ohmic losses. As a consequence Biermann's battery could no longer be considered as driving mechanism behind the strong magnetic fields of rotating stars, but could only provide seed fields for a dynamo.

Recently Biermann's battery has experienced a renaissance as provider of magnetic fields in the central tori of active galactic nuclei. The joint action of the rapid (Keplerian) differential rotation of these objects with fast outflow motions (and in some cases dynamo action) was considered an attractive mechanism for the production and spreading of magnetic fields in galaxies (e.g. Lesch et al. 1989; Chakrabarti 1991; Chakrabarti et al. 1994; Andreyasan 1996).
'Batteries' driven by gravitational-electromagnetic coupling have also been studied in the literature. Thorne et al. (1986) considered the model problem of a rotating black hole in vacuum, threaded by a stationary electric field. The coupling between gravitomagnetic potential of the hole and the electric field requires a stationary magnetic field. The electric field was, however, assumed to be due to a charged black hole, which is probably not very relevant in the astrophysical context. Opher \& Wichoski (1997) showed that nonminimal gravitational-electromagnetic coupling in protogalaxies could generate magnetic fields of $\sim 10^{-6}$ Gauss.

In the present paper I combine both Biermann-type battery effects with gravitomagnetic effects. The classical derivations of Biermann (1950) and Mestel \& Roxburgh (1962) are repeated using a new set of equations governing an inviscid (but resistive) plasma in the vicinity of a Kerr black hole, which have been derived by Khanna (1998) within the $3+1$ split of the Kerr metric. This set of equations contains a generalized Ohm's law, which is fundamental for the study of battery effects. I obtain a general relativistic expression for the 'Impressed Electric Field' (IEF), which is due to the electron partial pressure in a plasma in the vicinity of a rotating black hole. I show that, in axisymmetry, the gravitomagnetic contributions to the IEF are not curl-free and therefore drive currents and generate magnetic fields. The total IEF is likely to be rotational in general.

The metric has signs $(-+++)$ and $c=1$. Vectors and tensors in 3-dimensional space are denoted by arrows (e.g. 
$\vec{v}$ and $\stackrel{\leftrightarrow}{T}$ ) and covariant derivatives are indicated by vertical bars, e.g. $\nabla_{j} \beta^{i} \equiv \beta_{\mid j}^{i}$.

\section{A PLASMA BATTERY IN THE KERR METRIC}

In this section I will first review the $3+1$ split of the Kerr metric. Secondly, the equation of motion and the generalized Ohm's law will be combined to find an expression for the IEF that drives currents in a quasi-neutral plasma (quasi-neutral in the plasma rest frame) surrounding a rotating black hole.

\subsection{The $3+1$ split of the Kerr metric}

In the $3+1$ split of the Kerr metric (Thorne et al. 1986) spacetime is split into a family of three dimensional differentially rotating hypersurfaces of constant time with internal curvature. These hypersurfaces of constant time can be mentally collapsed into a single 3-dimensional 'absolute space' in which time is globally measured by the Boyer-Lindquist coordinate $t$. Physics is described in absolute space by locally non-rotating fiducial observers (FIDOs) with respect to their local proper time $\tau$ in their locally flat frames. The line element of the Kerr metric in $3+1$ notation is given by

$$
\begin{aligned}
d s^{2} & =g_{\alpha \beta} d x^{\alpha} d x^{\beta} \\
& =-\alpha_{\mathrm{g}}^{2} d t^{2}+h_{j k}\left(d x^{j}+\beta^{j} d t\right)\left(d x^{k}+\beta^{k} d t\right)
\end{aligned}
$$

where the lapse function is identified with the gravitional redshift

$\alpha_{\mathrm{g}} \equiv(d \tau / d t)_{\mathrm{FIDO}}=\frac{\rho}{\Sigma} \sqrt{\Delta}$,

the shift functions are the components of the gravitomagnetic potential $\vec{\beta}$ with

$\beta^{r}=\beta^{\theta}=0, \quad \beta^{\phi} \equiv-\omega=-(d \phi / d t)_{\mathrm{FIDO}}=-\frac{2 a M r}{\Sigma^{2}}$,

which describes the differential rotation of the FIDOs relative to distant observers. The components of the 3-metric $\stackrel{\leftrightarrow}{h}$ are

$h_{r r}=\frac{\rho^{2}}{\Delta}, \quad h_{\theta \theta}=\rho^{2}, \quad h_{\phi \phi}=\tilde{\omega}^{2}, \quad h_{j k}=0$ if $j \neq k$.

Note that $h_{j k}=g_{j k}$ but $h^{j k}=g^{j k}+\beta^{j} \beta^{k} / \alpha_{\mathrm{g}}^{2}$. The metric functions appearing here are defined as

$$
\begin{aligned}
\Delta & \equiv r^{2}-2 M r+a^{2}, \quad \rho^{2} \equiv r^{2}+a^{2} \cos ^{2} \theta, \\
\Sigma^{2} & \equiv\left(r^{2}+a^{2}\right)^{2}-a^{2} \Delta \sin ^{2} \theta, \quad \tilde{\omega} \equiv(\Sigma / \rho) \sin \theta .
\end{aligned}
$$

The parameters of the black hole are its mass $M$ and angular momentum $J$, which define the Kerr parameter $a \equiv J / M$.

The FIDO-measured velocities are

$v^{j}=\frac{1}{\alpha_{\mathrm{g}}}\left(\frac{d x^{j}}{d t}+\beta^{j}\right)$, e.g. $v^{\phi}=\frac{\Omega-\omega}{\alpha_{\mathrm{g}}}$.

The physical velocity components $v^{\hat{j}}$ follow by multiplcation with $\left(h_{j j}\right)^{1 / 2}$.

\subsection{Battery theory}

In a non-magnetized plasma, currents are driven by electric fields and the 'impressed electric field'. If the IEF possesses a potential, it may be cancelled by an electrostatic field and no current will flow. The IEF is included in a generalized Ohm's law, which is the base of battery theory.

If the plasma is 'cold' (pressure and internal energy of the components $\ll$ mass energy density) and quasi-neutral in its rest frame the generalized Ohm's law in the MHD limit is given by (Khanna 1998)

$\vec{j}=\sigma \gamma(\vec{E}+\vec{v} \times \vec{B})-\frac{\sigma}{e n}(\vec{j} \times \vec{B})+\frac{\sigma}{e n \alpha_{\mathrm{g}}} \vec{\nabla}\left(\alpha_{\mathrm{g}} p_{\mathrm{e}}\right)$.

Here $\vec{j}, \vec{E}, \vec{B}, \vec{v}$ and $\gamma$ have their usual meaning, but are measured locally by FIDOs. $n, p_{\mathrm{e}}$ and $\sigma$ are electron density, electron pressure and plasma conductivity, respectively, defined in the plasma rest frame (note that I do not distinguish between electron, ion and plasma rest frame. This is because treating a plasma as single fluid implies that electrons and ions have non-relativistic bulk velocities in the plasma rest frame; Khanna 1998). The 'impressed electric field'

$\vec{E}^{(i)}=\frac{\vec{\nabla}\left(\alpha_{\mathrm{g}} p_{\mathrm{e}}\right)}{e n \gamma \alpha_{\mathrm{g}}}$

can be re-expressed with the aid of the equation of motion to see, if it possesses a potential or not. To be correct, it is $\alpha_{\mathrm{g}} \vec{E}^{(i)}$ that matters, since the curl of $\alpha_{\mathrm{g}} \vec{E}$ appears in Faraday's law (see below).

The equation of motion for a 'cold' and quasi-neutral plasma (then the charge density measured by FIDOs is $\rho_{\mathrm{c}}=$ $\vec{v} \cdot \vec{j}$ ) has the form (Khanna 1998)

$$
\begin{aligned}
\rho_{\mathrm{m}} \gamma \frac{d(\gamma \vec{v})}{d \tau} & =\rho_{\mathrm{m}} \gamma^{2} \vec{g}+\rho_{\mathrm{m}} \gamma^{2} \stackrel{\leftrightarrow}{H} \cdot \vec{v}-\frac{1}{\alpha_{\mathrm{g}}} \vec{\nabla}\left(\alpha_{\mathrm{g}} p\right) \\
& +(\vec{v} \cdot \vec{j}) \vec{E}+\vec{j} \times \vec{B}
\end{aligned}
$$

with the energy density of the plasma defined in the plasma rest frame $\rho_{\mathrm{m}} \approx n_{\mathrm{i}} m_{\mathrm{i}}$ and $n_{\mathrm{i}}$ is the ion density. The total time derivative in the FIDO frame is defined as

$\frac{d}{d \tau} \equiv \frac{1}{\alpha_{\mathrm{g}}}\left[\frac{\partial}{\partial t}+\left(\alpha_{\mathrm{g}} \vec{v}-\vec{\beta}\right) \cdot \vec{\nabla}\right]$.

The total plasma pressure is given by

$$
\begin{aligned}
p & \equiv p_{\mathrm{i}}+p_{\mathrm{e}}=n_{\mathrm{i}} k T+\left(n_{\mathrm{e}} k T+\frac{b}{3} T^{4}\right) \\
& =\left(\frac{n_{\mathrm{i}}}{n_{\mathrm{e}}}+1\right) p_{\mathrm{e}}-\frac{n_{\mathrm{i}}}{n_{\mathrm{e}}} \frac{b}{3} T^{4} .
\end{aligned}
$$

Note that the radiation pressure appears as part of the electron partial pressure. This is because it is assumed that the interaction between radiation field and plasma is dominated by Compton scattering. Secondly $p \equiv p_{\mathrm{i}}+p_{\mathrm{e}}$ is a definition from the two-component plasma theory, which the equations used here are based upon. Therefore photons are not treated as a third component (effects of the radiation pressure will not be discussed in detail anyway). For a quasineutral plasma $\left(Z n_{\mathrm{i}} \approx n_{\mathrm{e}} \equiv n\right)$ Eq. (11) becomes

$p_{\mathrm{e}} \approx \frac{Z}{Z+1}\left(p+\frac{b}{3 Z} T^{4}\right)$

and the IEF is given by

$\vec{E}^{(i)} \approx \frac{Z}{e n \gamma \alpha_{\mathrm{g}}(Z+1)}\left(\vec{\nabla}\left(\alpha_{\mathrm{g}} p\right)+\frac{b}{3 Z} \vec{\nabla}\left(\alpha_{\mathrm{g}} T^{4}\right)\right)$. 
Eliminating $\vec{\nabla}\left(\alpha_{\mathrm{g}} p\right) / \alpha_{\mathrm{g}}$ with the aid of Eq. (9) yields

$$
\begin{aligned}
\vec{E}^{(i)} \approx & \frac{m_{\mathrm{i}}}{(Z+1) e}\left(\gamma \vec{g}+\stackrel{\leftrightarrow}{H} \cdot(\gamma \vec{v})-\frac{d(\gamma \vec{v})}{d \tau}\right) \\
& +\frac{Z(\vec{j} \times \vec{B}+(\vec{v} \cdot \vec{j}) \vec{E})}{(Z+1) e n \gamma}+\frac{b \vec{\nabla}\left(\alpha_{\mathrm{g}} T^{4}\right)}{3(Z+1) e n \gamma \alpha_{\mathrm{g}}}
\end{aligned}
$$

This expression shows that, for relativistic plasma velocities, even gravity will drive currents, unless $\gamma$ is a function of $\alpha_{\mathrm{g}}$ only (since $\vec{g}=-\vec{\nabla} \ln \alpha_{\mathrm{g}}$ ) or a constant. For most practical purposes, however, $\gamma \approx 1$, even close (but not too close) to a rotating black hole, and the IEF due to gravity will be balanced by an electrostatic field. I note in passing that the IEF due to radiation pressure will also be rotational unless $\gamma n$ is a function of the radiation pressure. This topic will, however, not be pursued any further in this paper.

Inserting $\vec{E}^{(i)}$ into Ohm's law yields

$$
\begin{aligned}
\vec{j} & \approx \sigma \gamma\left(\left(\frac{Z(\vec{v} \cdot \vec{j})}{(Z+1) e n \gamma}+1\right) \vec{E}+\vec{v} \times \vec{B}\right) \\
& +\frac{\sigma \gamma m_{\mathrm{i}}}{(Z+1) e}\left(\gamma \vec{g}+\stackrel{\leftrightarrow}{H} \cdot(\gamma \vec{v})-\frac{d(\gamma \vec{v})}{d \tau}\right) \\
& -\frac{\sigma}{(Z+1) e n} \vec{j} \times \vec{B}+\frac{\sigma b \vec{\nabla}\left(\alpha_{\mathrm{g}} T^{4}\right)}{3(Z+1) e n \alpha_{\mathrm{g}}}
\end{aligned}
$$

The magnetic field associated with the current described by Eq. (15) is given by Ampère's law

$\vec{\nabla} \times\left(\alpha_{\mathrm{g}} \vec{B}\right)=\left(\frac{\partial}{\partial t}-\mathcal{L}_{\vec{\beta}}\right) \vec{E}+4 \pi \alpha_{\mathrm{g}} \vec{j}$.

Note that the sources of magnetic fields are not only currents and the displacement current, but also the coupling between the gravitomagnetic potential and electric fields. This has interesting consequences, which show up as 'battery effects' (Thorne et al. 1986) or dynamo effects (Khanna \& Camenzind 1996a,b). The generation of magnetic fields, however, requires, as usual, a rotational 'renormalized' electric field, as demanded by Faraday's law

$\vec{\nabla} \times\left(\alpha_{\mathrm{g}} \vec{E}\right)=-\left(\frac{\partial}{\partial t}-\mathcal{L}_{\vec{\beta}}\right) \vec{B}$

The Lie-derivative is defined as, e.g. $\mathcal{L}_{\vec{\beta}} \vec{B} \equiv(\vec{\beta} \cdot \vec{\nabla}) \vec{B}-$ $(\vec{B} \cdot \vec{\nabla}) \vec{\beta}$. It is straight forward to show that, since $\vec{\nabla} \cdot \vec{B}=$ $0, \mathcal{L}_{\vec{\beta}} \vec{B}=-\vec{\nabla} \times(\vec{\beta} \times \vec{B})$. Faraday's law can therefore be written as

$\vec{\nabla} \times\left(\alpha_{\mathrm{g}} \vec{E}+\vec{\beta} \times \vec{B}\right)=-\frac{\partial \vec{B}}{\partial t}$.

This means that stationary magnetic field requires $\alpha_{\mathrm{g}} \vec{E}+$ $\vec{\beta} \times \vec{B}=-\vec{\nabla} \Phi$, where $\Phi$ is a scalar potential. Maxwell's equations are completed by

$\vec{\nabla} \cdot \vec{B}=0 \quad$ and $\quad \vec{\nabla} \cdot \vec{E}=4 \pi \rho_{\mathrm{c}}$.

\subsubsection{The gravitomagnetic battery}

Let us, for the moment, restrict ourselves to the generation of magnetic field by the electron partial pressure, i.e. let us drop the terms containing $\vec{B}$ and neglect the electric force term $(\vec{v} \cdot \vec{j}) \vec{E}$ in Eq. (15). Leaving aside contributions from $\gamma \vec{g}$ and from radiation pressure, currents will be driven by the rotational parts of $\vec{E}$, the relativistic equivalent of the classical IEF
$\vec{E}_{\text {class }}^{(i)}=-\frac{m_{\mathrm{i}}}{(Z+1) e}(\vec{v} \cdot \vec{\nabla})(\gamma \vec{v})$,

which is responsible for Biermann-type batteries, the gravitomagnetic IEF

$\vec{E}_{\mathrm{gm}}^{(i)}=\frac{m_{\mathrm{i}}}{(Z+1) e \alpha_{\mathrm{g}}}(\vec{\beta} \cdot \vec{\nabla}+\vec{\nabla} \vec{\beta} \cdot)(\gamma \vec{v})$

and the IEF due to time-dependent plasma motion

$\vec{E}_{\partial t}^{(i)}=-\frac{m_{\mathrm{i}}}{(Z+1) e \alpha_{\mathrm{g}}} \frac{\partial(\gamma \vec{v})}{\partial t}$.

$\alpha_{\mathrm{g}} \vec{E}_{\partial t}^{(i)}$ will contribute to the current, if $\vec{\nabla} \times(\gamma \vec{v}) \neq 0$, i.e. if there are non-stationary vortices in the fluid.

In the following I will analyse $\alpha_{\mathrm{g}} \vec{E}_{\text {class }}^{(i)}$ and $\alpha_{\mathrm{g}} \vec{E}_{\mathrm{gm}}^{(i)}$ for $\vec{v}=v^{r} \vec{e}_{r}+v^{\phi} \vec{e}_{\phi}$ and discuss under which circumstances those IEFs possess a potential: From $\alpha_{\mathrm{g}} \vec{E}_{\text {class }}^{(i)}$ we have

$$
\begin{aligned}
-\left(\alpha_{\mathrm{g}} \vec{v} \cdot \vec{\nabla}\right)(\gamma \vec{v})=-\left(\alpha_{\mathrm{g}} v^{i}\right)\left(\gamma v^{j}\right)_{\mid i} \vec{e}_{j} \\
\quad=\alpha_{\mathrm{g}} \gamma\left(v^{\phi}\right)^{2} \tilde{\omega} \vec{\nabla} \tilde{\omega} \\
\quad-\alpha_{\mathrm{g}}\left(v^{r}\left(\gamma v^{r}\right)_{, r}+\gamma\left(v^{r}\right)^{2}\left(\ln \sqrt{h_{r r}}\right)_{, r}+v^{\phi}\left(\gamma v^{r}\right)_{, \phi}\right) \vec{e}_{r} \\
\quad+\alpha_{\mathrm{g}} \gamma\left(v^{r}\right)^{2}\left(\ln \sqrt{h_{r r}}\right)_{, \theta} \vec{e}_{\theta} \\
\quad-\alpha_{\mathrm{g}}\left(v^{r}\left(\gamma v^{\phi}\right)_{, r}+2 \gamma v^{\phi} v^{r}(\ln \tilde{\omega})_{, r}+v^{\phi}\left(\gamma v^{\phi}\right)_{, \phi}\right) \vec{e}_{\phi} \cdot(23)
\end{aligned}
$$

Though it is unlikely that this expression should be irrotational in general, it is not possible to make statements, unless $v^{r}$ and $v^{\phi}$ are known explicitly. If, however, $v^{r}=0$ in an axisymmetric problem, only the first term on the r.h.s. remains. This is the relativistic equivalent of Biermann's IEF in a star. This expression is irrotational only if $\alpha_{\mathrm{g}} \gamma\left(v^{\phi}\right)^{2}$ is a function of $\tilde{\omega}$ alone, i.e. if, for large radii, $\gamma\left(v^{\phi}\right)^{2}$ is constant on cylinders. This is unlikely and certainly not possible within the ergosphere of the black hole; but there $v^{r} \neq 0$ anyway. The relativistic equivalent of Biermann's battery contributes therefore also around black holes. The function part of $\alpha_{\mathrm{g}} \vec{E}_{\mathrm{gm}}^{(i)}$ is

$$
\begin{aligned}
(\vec{\beta} \cdot \vec{\nabla} & +\vec{\nabla} \vec{\beta} \cdot)(\gamma \vec{v})=\left(\beta^{i}\left(\gamma v^{j}\right)_{\mid i}+\gamma \beta^{i \mid j} v_{i}\right) \vec{e}_{j} \\
& =-\gamma v^{\phi} \tilde{\omega}^{2} \vec{\nabla} \omega-\omega\left(\left(\gamma v^{r}\right)_{, \phi} \vec{e}_{r}+\left(\gamma v^{\phi}\right)_{, \phi} \vec{e}_{\phi}\right) .
\end{aligned}
$$

In axisymmetry $\alpha_{\mathrm{g}} \vec{E}_{\mathrm{gm}}^{(i)}$ is clearly rotational, unless some freak $\gamma$ should manage to make $\gamma v^{\phi} \tilde{\omega}^{2}$ a function of $\omega$ alone. Only if $v^{\phi}$ is non-axisymmetric, the gravitomagnetic IEF drives toroidal currents. With Eqs. 23) and (24), it seems obvious that $\alpha_{\mathrm{g}}\left(\vec{E}_{\partial t}^{(i)}+\vec{E}_{\text {class }}^{(i)}+\vec{E}_{\mathrm{gm}}^{(i)}\right)$ will be rotational in general. Note, however, that if axisymmetry is imposed the toroidal component of the total IEF (Eq. 8) is zero, which means that, even though $\alpha_{\mathrm{g}}\left(\vec{E}_{\partial t}^{(i)}+\vec{E}_{\text {class }}^{(i)}\right)$ may have a rotational toroidal component, no toroidal currents will be driven. In axisymmetry poloidal magnetic fields can not be generated by the IEF. Once a toroidal magnetic field has been induced, however, the gravitomagnetic term $\mathcal{L}_{\vec{\beta}} \vec{E}$ will generate a poloidal magnetic field (Khanna \& Camenzind 1996a; and see below).

\subsubsection{The equilibrium magnetic field}

We can now be confident that, but for pathological velocity fields, the gravitomagnetic battery will generate magnetic fields. The important question that remains to be answered is how strong these fields can become. In the case of Biermann's battery, Mestel and Roxburgh (1962) have shown 
that the fields are limited to very low field strength, and are only significant as seed fields for a dynamo. In a selfconsistent picture the limitation of the magnetic field is not due to Ohmic dissipation, but occurs at much lower level due to the back reaction of the magnetic field on the velocity field.

The evolution of the magnetic field generated by the battery is given by Eq. (18). Eliminating $\vec{E}$ with the aid of Eq. 15) (neglecting rotational contributions from $\gamma \vec{g}$ and radiation pressure) and using Eq. (16) we get the generalized MHD induction equation

$$
\begin{aligned}
\frac{\partial \vec{B}}{\partial t} & =\vec{\nabla} \times\left(\left(\frac{\alpha_{\mathrm{g}} \vec{v}}{\kappa}-\vec{\beta}\right) \times \vec{B}\right) \\
& -\vec{\nabla} \times \frac{\eta}{\gamma \kappa}\left(\vec{\nabla} \times\left(\alpha_{\mathrm{g}} \vec{B}\right)-\left(\frac{\partial}{\partial t}-\mathcal{L}_{\vec{\beta}}\right) \vec{E}\right) \\
& -\vec{\nabla} \times \frac{\alpha_{\mathrm{g}}}{(Z+1) e \kappa}\left(\frac{\vec{j} \times \vec{B}}{n \gamma}+m_{\mathrm{i}}\left(\frac{d}{d \tau}-\stackrel{\leftrightarrow}{H} \cdot\right)(\gamma \vec{v})\right)
\end{aligned}
$$

where $\kappa=1+Z(\vec{v} \cdot \vec{j}) /(Z+1)$ en $\gamma$. The magnetic diffusivity has been introduced as $\eta=c^{2} /(4 \pi \sigma)$. This equation merits some discussion: The first two lines (if $\kappa=1$ ) are the MHD induction equation, which one derives with the standard relativistic Ohm's law. In special relativity there is no $\mathcal{L}_{\vec{\beta}} \vec{E}$ and Cowling's theorem against axisymmetric dynamo action (Cowling 1934) holds. The presence of $\mathcal{L}_{\vec{\beta}} \vec{E}$ in the context of the Kerr metric states that Cowling's theorem does, in principle, not hold in the vicinity of a rotating black hole. In the axisymmetric standard-Ohm-case and if the displacement current is neglected, $\vec{E}$ can be eliminated and the equation can be split into poloidal and toroidal parts to yield two coupled partial differential equations with $\mathcal{L}_{\vec{\beta}} \vec{E}$ (expressed by the poloidal and toroidal magnetic fields) appearing as source term for the poloidal magnetic field (Khanna \& Camenzind 1996a).

In the general case, Eq. (25) is not of much practical use, since $\vec{E}$ and $\vec{j}$ can not be eliminated. But it serves for discussion of the processes. The equation describes the joint action of battery and gravitomagnetic dynamo. Although numerical simulations of the kinematic axisymmetric gravitomagnetic dynamo with standard Ohm's law have so far failed to produce growing or stationary modes (Khanna \& Camenzind 1996b; but see also Núñez 1997), it may well be that they appear with the aid of the IEF.

In stars the limitation of the thermal battery is due to modification of $\vec{v}$ by Lorentz forces. This has a quadratic effect on $(\vec{v} \cdot \vec{\nabla}) \vec{v}$ so that ultimately $\vec{\nabla} \times \vec{E}=0$ can be achieved. The gravitomagnetic battery terms are linear in $\vec{v}$ so that the approach of the IEF plus limiting terms to an irrotational state can be expected to take place much slower, which should result in a higher value of the asymptotic magnetic field.

Quantitative analytic estimates of the asymptotic state in this even more complicated system than the one considered by Mestel \& Roxburgh (1962) for the Biermann battery are very hard to achieve and can only be very crude. The solution of the battery/dynamo problem in Kerr metric has to be postponed until a numerical treatment of MHD is feasible.

\section{CONCLUSION}

It has been shown that in a quasi-neutral MHD-plasma, which is in axisymmetric motion around a rotating black hole, the gravitomagnetic 'Impressed Electric Field' is rotational and will drive currents, thus generating a magnetic field. For the general case the gravitomagnetic IEF is likely to be rotational, but explicit velocity fields will have to be studied. The same holds for the total IEF. Poloidal magnetic fields can only be induced, if the electron partial pressure is non-axisymmetric.

In Kerr metric the battery problem can not be treated separately from the gravitomagnetic dynamo problem. It is therefore very unlikely that reliable analytical estimates of the equilibrium field strength of the system can be achieved. It is argued that, since the gravitomagnetic field of the rotating black hole can not be quenched by Lorentz forces (braking of the hole's rotation by battery generated fields is negligible) and the gravitomagnetic battery terms are only linearly dependent on the velocity field of the plasma, the equilibrium field should be stronger than in the case of the classical Biermann battery.

The expression for the total IEF reveals the fact that, for relativistic motions with spatially variable Lorentz factor, even gravity will make rotational contributions to the IEF, i.e. it would drive currents even in Schwarzschild metric. Also time-dependent vortices of the velocity field drive currents.

This work is based on the generalized Ohm's law for a quasi-neutral plasma in the MHD limit. In a charged plasma there are 'acceleration currents' and 'gravitomagnetic currents', which might give qualitatively new results. On timescales of the electron collision time, 'current acceleration' terms become important, which might even introduce additional axisymmetric dynamo effects (Khanna 1998).

Radiation pressure does also contribute to the rotational part of the IEF. This has not been discussed in detail, but could be significant in the hot radiative region close to the horizon. In this region, the single-fluid approach of MHD might break down alltogether (Khanna 1998) and the conlusions drawn here would have to be checked in a multi-fluid model.

\section{ACKNOWLEDGEMENTS}

I thank Max Camenzind, Jochen Peitz, Stefan Spindeldreher and Markus Thiele for fruitful discussions. This work is supported by the Deutsche Forschungsgemeinschaft (SFB 328).

\section{REFERENCES}

Andreyasan, R.R., 1996, Astrophysics 39, No. 1, 58

Biermann, L., 1950, Z. Naturforschg. 5a, 65

Chakrabarti, S.K., 1991, MNRAS 252, 246

Chakrabarti, S.K., Rosner, R., Vainshtein, S.I., 1994, Nature 368, 434

Cowling T.G., 1934, MNRAS 94, 39

Khanna, R., 1998, MNRAS 294, 673; astro-ph/9803088

Khanna, R., Camenzind, M., 1996a, A\&A 307, 665

Khanna, R., Camenzind, M., 1996b, A\&A 313, 1028

Lesch, H., Crusius, A., Schlickeiser, R., Wielebinski, R., 1989, A\&A 217, 99 
Mestel, L., Roxburgh, J.W., 1962, ApJ 136, 615

Núñez, M., 1997, Phys. Rev. Lett., 79, 796

Opher, R., Wichoski, U.F., 1997, Phys. Rev. Lett. 78, 787-790

Thorne, K.S., Price, R.H., Macdonald, D.A., Suen, W.-M., Zhang, X.-H., 1986, in: Black Holes: The Membrane Paradigm, Thorne, K.S., Price, R.H., Macdonald, D.A., (Eds.), Yale

Univ. Press, New Haven, pp. 67-120 